\title{
Subject Index Vol. 2, 1996
}

Abstinence 163 Addiction 163, 185 Admission modalities 213 AIDS 169

prevention 109 Alcohol 73, 102

dependence 29,94

dependency 129

withdrawal 29, 83 Alcoholism 17, 140, 156

treatment 94

American drug control policies 177 ASI 124 Assertiveness 73 Assessment 129

Benzodiazepine addiction 53

- $\quad$ dependence syndrome 53

British 185

Carbohydrate-deficient transferrin 140,

156 Carcinoma 156 CIDI 124 CIDI-SAM 124 Clinical trial 94 Cognitive dysfunction 83

Comorbidity 11,24,29,36,147,213

- $\quad$ and crime 3

Criminal responsibility 3

Culture 78

Depression 24, 29

Depressive disorders 17

Disinhibition 73

Disposition 213

Dissociative Experience Scale (DES) 53

Drug abuse-related death 169

addiction 147

addicts 109 Drugs 219

Emergency service 213 Epidemiology 169

- $\quad$ of mental disorders 36

Ethnic variations 78

European drug control policies 177 Executive function 83

FMP 53 Follow-up 147 Forensic psychiatry 3

$\gamma$-Glutamyltransferase 140

Health policy 219 Heroin 177

addiction 49

injecting 194

prescribing 185,194

prohibition 177 History 219

HIV-1 prevalence 169

ICD-10 53

Inpatient psychotherapy 53 Intensive care unit 156 Interview 124

Markers 140 
Mean corpuscular volume 140 Medical use of heroin 177 Mental absorption 102 Methadone maintenance 49 Millon Behavioral Health Inventory Minority peoples 78 Morphine 208 -, side effects 208

- $\quad$ substitution treatment 208

Mortality 49

Multi-axial forensic assessment 3

Northern areas 78

Opiate dependence 194 Opioid 163

Personality 102

- $\quad$ disorders 147

Phobic disorders 17

Prefrontal cortex 83

Prevalence 11, 36

Psychiatric comorbidity 17

Psychopathology 17

Psychotherapeutic outcome 53

Psychotherapy 147

Rating scales 129 Religious norms 78 Residential treatment 147

Saami 78 SCAN 124 Schizophrenia 11 SCL-90 53 Screening 94, 129 Social behavior 73

Stimulant dependence 24 Substance abuse 11

- and crime 3

- - dependence 36

abusers 213 Symptom checklist 124

Therapy 29

United Kingdom 194

Vestibular organs 163

228 